\title{
路易斯碱一硼自由基在磞化反应、自由基催化和还原反应中的应用
}

\author{
靳继康夏慧敏张凤莲汪义丰* \\ (中国科学技术大学化学系 合肥 230026)
}

\begin{abstract}
摘要 自由基反应具有高效迅捷、选择性优良及官能团容忍性好等优点, 是构建有机化合物的有效策略和方式之一. 路易斯碱-嗍自由基具有独特的结构和反应性能, 在有机合成中表现出了良好的应用潜力. 总结了我们课题组在路易 斯碱一嗍自由基促进的有机分子转化方面取得的一些进展. 研究成果主要包括以下三个方面: 路易斯碱-硼自由基促进 的硼化反应、路易斯碱-硼自由基催化反应，以及路易斯碱-硼自由基引发的还原反应. 这些反应具有条件温和，官能团 容忍性好, 产率高, 化学、区域选择性好等优点.

关键词 路易斯碱-硼自由基; 自由基硼化反应; 自由基催化反应; 自由基还原反应; 自由基化学
\end{abstract}

\section{Lewis-Base Boryl Radicals Enabled Borylation, Radical Catalysis and Reduction Reactions}

\author{
Jin, Jikang Xia, Huimin Zhang, Fenglian Wang, Yifeng* \\ (Department of Chemistry, University of Science and Technology of China, Hefei 230026)
}

\begin{abstract}
Free radical reactions represent an efficient and significant tool to construct organic molecules by taking advantages of the high-efficiency, remarkable selectivity and good functional groups tolerance. Lewis-base boryl radicals are a class of species that possess unique structures and chemical reactivity, and a variety of synthetic applications have been developed. This account summarizes the research advances in this research field mainly contributed by our group. The results include Lewis-based boryl radicals enabled borylation reactions, Lewis-based boryl radicals-catalyzed new reactions, and Lewis-based boryl radicals promoted reduction reactions. These reactions feature mild reaction conditions, good functional groups compatibility, high yields, and excellent chemo-, regio-, and stereo-selectivities.

Keywords Lewis-base boryl radicals; radical borylation reactions; radical catalysis; radical reduction reactions; free radical chemistry
\end{abstract}

化学键的构建和断裂是合成化学的基础, 是创造新 物质的基石和源泉. 路易斯碱-硼自由基作为一类具有 独特结构和反应性能的活性物种, 为多种化学键的高效 构建和断裂提供了新的策略和途径. 20 世纪 80 年代, Roberts ${ }^{[1]}$ 在该领域做出了开创性的工作, 其课题组详细 研究了胺-嗍自由基和膦-嗍自由基的生成、结构以及反 应性能, 在自由基脱卤和氢转移方面取得了一些重要的 研究进展. 但是, 该方面的研究并未引起人们的广泛关 注. 直到 2008 年, Curran 及其合作者 ${ }^{[2]}$ 开发了氮杂环卡 宾-硼自由基 $\left(\mathrm{NHC}-\mathrm{BH}_{2} \bullet\right)$, 并实现了多种类型的合成转 化, 包括黄原酸酯的脱氧反应、卤代烃的自由基还原反

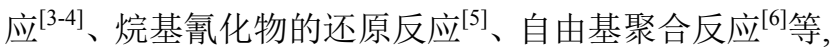
在一定程度上丰富了路易斯碱一嗍自由基的研究内容. 但是，该方面的研究仍存在以下几个缺点：(1)反应类型 过于单一, 路易斯碱一硼烷仅作为有毒的 $n-\mathrm{Bu}_{3} \mathrm{SnH}$ 替代 品, 实现了一系列的自由基还原反应; (2)原子经济性较 低，反应中路易斯碱-嗍烷部分常作为副产物被丢弃; (3)路易斯碱-嗍自由基的产生方式局限于算取路易斯 碱一嗍烷的氢原子; (4)难以构建有机硼化合物等. 因此, 探索嗍自由基多样性产生方式, 发展新的硼自由基前 体，并开发嗍自由基在有机合成中的应用，解决传统合 成方法存在的弊端, 在合成化学的研究中具有十分重要

\footnotetext{
* Corresponding author. E-mail: yfwangzj@ustc.edu.cn

Received May 7, 2020; revised May 19, 2020; published online May 27, 2020.

Project supported by the National Natural Science Foundation of China (Nos. 21672195, 21702201, 21971226) and the Fundamental Research Funds for the Central Universities (No. WK2060190082).

国家自然科学基金(Nos. 21672195, 21702201, 21971226)和中央高校基本科研业务费专项资金(No. WK2060190082)资助项目.
} 
的意义.

我们课题组近年来一直致力于开发路易斯碱一硼自 由基促进的新型的有机合成反应. 本文主要总结了我们 在硼自由基的产生, 及其在合成应用中取得的一些研究 进展, 主要分为以下三个部分: 硼自由基促进的硼化反 应、硼自由基催化反应、嗍自由基促进的还原反应.

\section{1 路易斯碱一嗍自由基促进的硼化反应研究进 展}

\section{1 路易斯碱-嗍自由基促进的嗍化/环化串联反应}

有机嗍化合物具有独特的物理和化学性能, 广泛应 用于诸多领域 ${ }^{[7]}$. 近年来, 多种高效构建有机硼化合物 的方法已经被开发和利用. 例如诺贝尔奖得主 Brown $^{[8]}$ 发展了一系列亲电性的硼试剂和烯烃、炔烃的加成反应, 实现了多种烷基硼和烯基嗍化合物的高效合成. Miyaura 及其合作者 ${ }^{[9]}$ 开发了亲核性的硼酸盐物种和芳 基卤代物的偶联反应，实现了芳基硼化合物的构建. 尽 管有机硼化合物的合成已经取得长足进步, 但是如何以 优良的化学、区域和立体选择性构建有机硼化合物, 尤 其是结构复杂的有机嗍化合物，仍具有较大挑战.

相较于亲电和亲核反应，自由基反应在反应性能、 效率、选择性和经济性等方面表现出一些优良的特 性 ${ }^{[10]}$. 可以设想, 如果能实现碳-碳不饱和体系的自由 基硼化反应，这将为有机硼化合物的构建开辟全新的思 路和途径. 开展这方面工作的主要挑战在于: (1)如何找 到合适的硼自由基前体，(2)如何开发硼自由基的新反 应，(3)如何设计合适的反应体系以保障链反应的高效传 递, (4)如何将嗍化产物转化为功能化的硼酸酯类化合 物.

基于上述考量, 我们课题组 ${ }^{[11]}$ 以氮杂环卡宾-硼烷 加合物 $\left(\mathrm{NHC}-\mathrm{BH}_{3}\right.$ ) 为硼自由基前体, 利用自由基串联反 应和极性反转策略, 率先开发了一例嗍自由基促进的 1,6-烯炔化合物的嗍化串联环化反应. 反应以优良的化 学、区域和立体选择性实现了烷基或烯基硼官能团取代 的六元环状化合物的合成(Scheme 1a) ${ }^{[12]}$. 基于实验结 果和机理研究结果, 对该反应提出了可能的反应机理 (Scheme 1b). 在此反应中, 自由基引发剂加热裂解, 㩲 取氮杂环卡宾-硼烷上的氢原子产生相应的嗍自由基中 间体 II ${ }^{[13]}$. 随后, 硼自由基 II 选择性地加成于芳环所共 轭的碳碳双键或碳碳三键生成相应的苄基自由基中间 体 III 或烯基自由基中间体 V. 接着, 6-exo 关环得到相 应的烯基 IV 或烷基自由基 VI 中间体. 这两种自由基中 间体具有亲核性, 从富电子的氮杂环卡宾-硼烷加合物 上瞳氢是不利的. 我们利用极性反转策略, 在体系中加 入硫醇催化剂, 可以有效促进氢原子转移反应, 从而还 (a) Radical borylation/cyclization cascade of 1,6-enynes

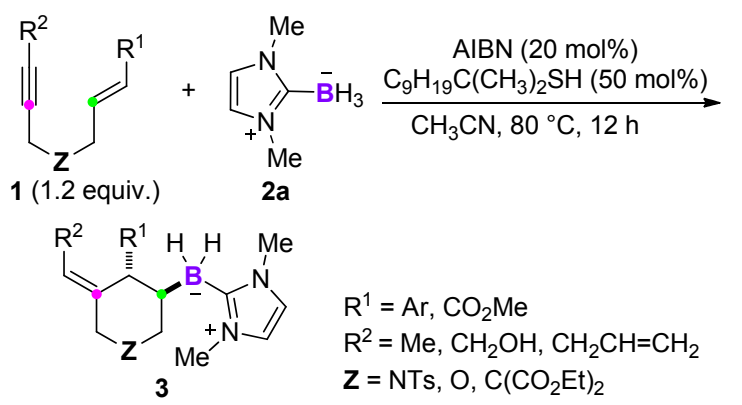

(single diastereomer)

16 examples, $29 \% \sim 92 \%$

2a (1.5 equiv)

$\mathrm{Me} \mathrm{H}, \mathrm{H}$ Ar $\mathrm{R}^{3}$

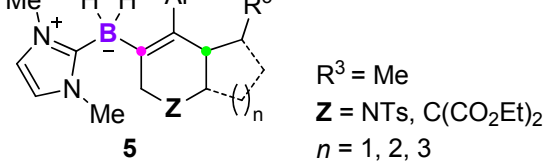

13 examples, $35 \% \sim 81 \%$

$n=1,2,3$

(b) Plausible mechanisms
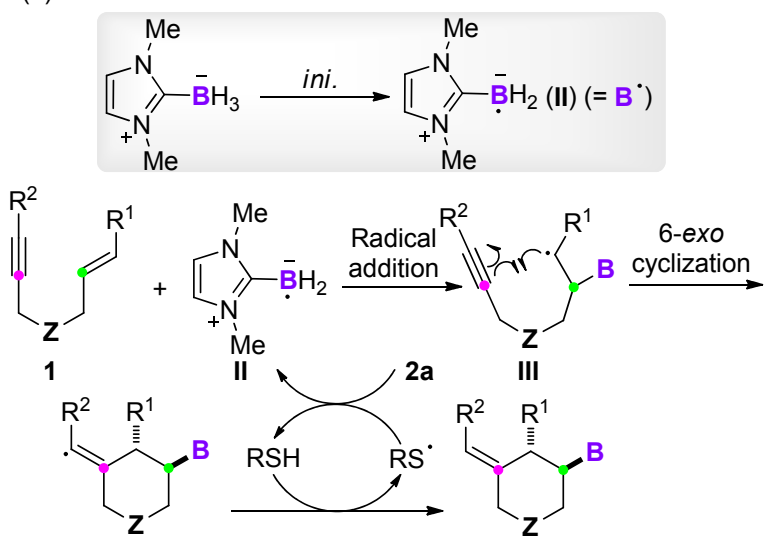

IV 3

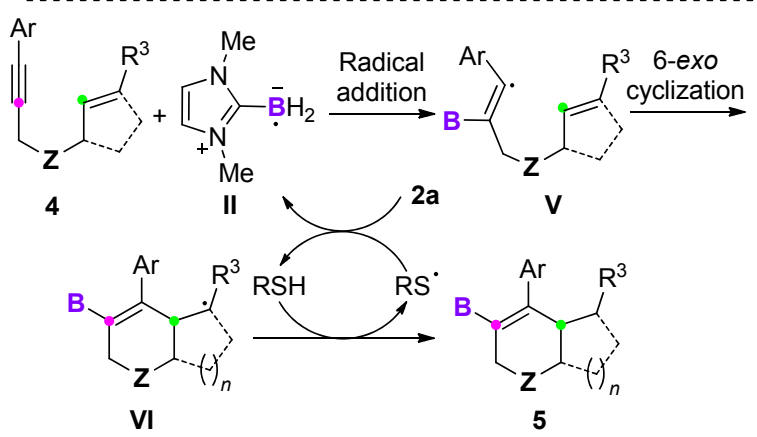

图式 1 1,6-烯炔的自由基硼化环化串联反应

Scheme 1 Radical borylation/cyclization cascade of 1,6-enynes

原 IV 或 VI 得到硼化环化产物 3 或 5 . 产生的硫自由基 具有亲电性，很容易瞳取氮杂环卡宾一硼烷加合物上的 氢原子 ${ }^{[3]}$, 重新生成硫醇和氮杂环卡宾-嗍自由基 II, 实 现了该串联环化反应的催化循环. 该硼自由基促进的串 
联环化反应, 为有机嗍化合物的可调控构建提供了一种 不同于以往的, 全新的策略和方法.

在此工作中, 我们对制得的嗍化产物进行了衍生化 和转化探究(Scheme 2). 烷基和烯基频哪醇硼酸酯在有 机合成中应用广泛. 使用 $2 \mathrm{~mol} / \mathrm{L} \mathrm{HCl}$ 水溶液和频哪醇 对 3a 和 5a 分别进行处理, 能够实现烷基 6 和烯基 9 频 哪醇嗍酸酯取代的六元环状化合物的高效合成 ${ }^{[14]}$. 使 用选择性氟试剂对 3a 和 $5 \mathrm{a}$ 进行氟化反应, 嗍原子上的 氢原子能够转化为氟原子. 已有文献报道, 此类嗍原子 被氟化的烯基硼试剂能够作为偶联试剂实现偶联反 应 ${ }^{[15]}$. 另外, 使用 $\mathrm{H}_{2} \mathrm{O}_{2}$ 和 $\mathrm{NaBO}_{3}$ 分别对 3a 和 $\mathbf{5 a}$ 进行 氧化, 能够得到相应的六元环醇 8 和六元环酮 $\mathbf{1 1}$ 产物.

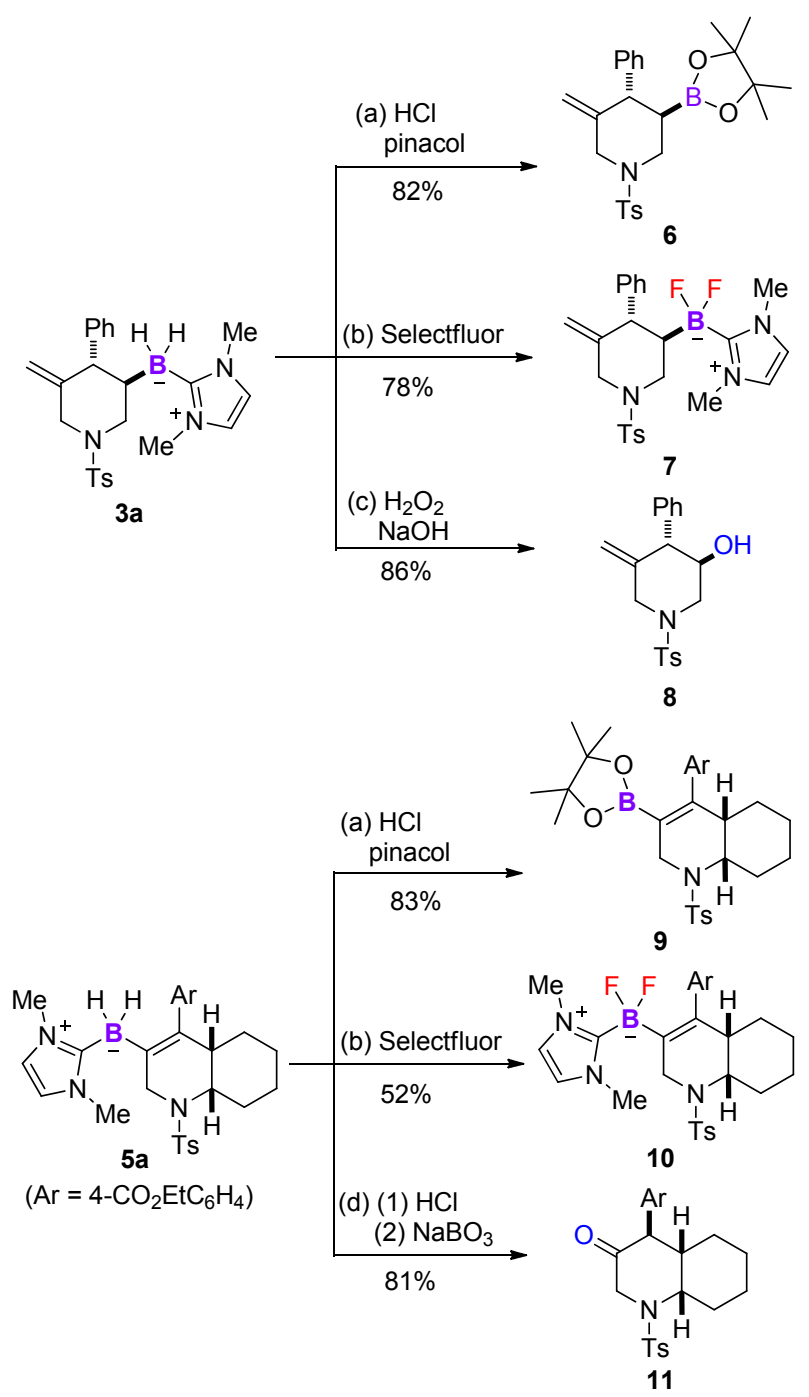

图式 2 嗍化产物的衍生化研究

Scheme 2 Transformations of borylated products

与此同时, Taniguchi 和 Curran 等 ${ }^{[16]}$ 开发了一例氮杂 环卡宾-硼自由基和具有特殊结构的 1,5-二炔化合物的 嗍化环化串联反应, 实现了氮杂环卡宾一嗍烷取代的五 元环并七元环产物的合成.
六元杂环和多环化合物广泛存在于天然产物、生物 活性分子和农药等 ${ }^{[17-18]}$. 由于 $\mathrm{C}-\mathrm{B}$ 键易于转化为多种 $\mathrm{C}-\mathrm{C}$ 键及 $\mathrm{C}-\mathrm{X}$ 键的优势 ${ }^{[19]}$, 发展高效的嗍官能团取 代的六元杂环和多环化合物的合成方法, 能够为六元杂 环和多环化合物的多样性合成及其后期衍生化提供一 种可行的策略. 2018 年, 我们课题组 ${ }^{[20]}$ 发展了一例 1,6二烯的自由基硼化环化串联反应，以优良的选择性实现 了氮杂环卡宾一嗍烷取代的六元杂环的高效合成 (Scheme 3a). 在此反应中, 氮杂环卡宾-硼自由基首先 以专一的化学和区域选择性加成于芳环或羰基所共轭 的碳碳双键, 进一步 6-exo 串联环化于分子内另一碳碳 双键. 最终, 实现了嗍官能团取代的六元杂环化合物的 构建. 非常有意思的是, 利用此串联环化反应能够实现 多种硼官能团取代的桥环和多环化合物的制备(Scheme 3b). 此外, 应用该方法, 我们还实现了抗抑郁药帕罗西 汀含嗍类似物 17 的高效合成. 含硼基团经进一步修饰, 最终成功转化为多种官能团取代的帕罗西汀类似物.

氮杂环化合物，尤其是环椫和喹唑啉酮，广泛存在 于多种天然产物和治疗性药物 ${ }^{17,21]}$. 因此, 发展高效构 建嗍官能团取代的环脒和喹唑啉酮化合物具有重要意 义. 在我们课题组前期工作的基础上, 2018 年, 我们 ${ }^{[22]}$ 开发了一例 $N$-烯丙基氰胺的嗍化环化串联反应，实现 了多种嗍官能团取代的环榺和喹唑啉酮化合物的高效 构建(Scheme 4). 在此反应中, 氮杂环卡宾-硼自由基以 优良的区域和化学选择性加成于芳环所共轭的碳碳双 键, 产生的碳自由基中间体进一步加成于分子内的 $N$ 氧基上得到相应的脒基自由基中间体，最终经箱氢或分 子内环化得到嗍取代的环脒化合物 23 或喹唑啉酮化合 物 25. 这一研究结果加深了我们对硼自由基的反应性 能的认识, 同时为具有生物活性的含氮杂环天然产物和 药物的制备提供了新思路.

\section{2 路易斯碱一硼自由基促进的硼氢化反应}

嗍氢化反应在有机合成中应用十分广泛，是高效构 建有机嗍化合物的一种重要方法 ${ }^{[8]}$. 近年来, 关于硼氢 化反应的研究已经取得了重要的进展 ${ }^{[23]}$, 但官能团兼 容性和反应的区域选择性方面仍存在一些问题和挑战. 因此，发展新的策略来实现与传统硼氢化反应完全不同 的选择性，具有十分重要的意义.

近年来, 多个课题组报道了利用自由基途径实现硼 氢化反应. 研究表明, 相较于传统的硼氢化方法, 嗍自 由基参与的硼氢化反应表现出不一样的反应特性. 例 如, 2018 年, 朱成建和谢劲课题组 ${ }^{[24]}$ 报道了一例亚胺的 自由基嗍氢化反应，实现了 $\alpha$-氨基嗍化合物的高效合 成. 非常有意思的是，该嗍自由基促进的硼氢化反应和 经典的亚胺硼氢化反应区域选择性相反. Taniguchi 及其 
(a) Radical borylative cyclization of 1,6-dienes<smiles>[R]C=CC[Z]([R2])([H])CC=C([R])[R]</smiles>

18 examples, $42 \% \sim 90 \%$

(b) Synthesis of boron-handled polycyclics (6 examples)

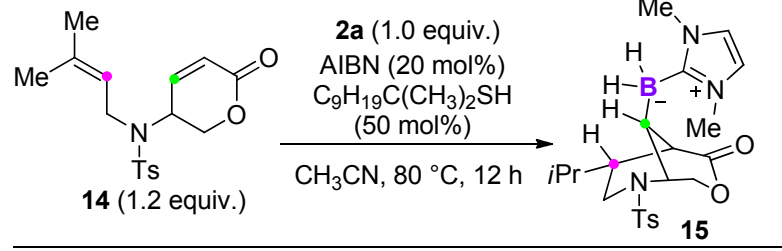

(c) Diversification of boryl-handled Paroxetine
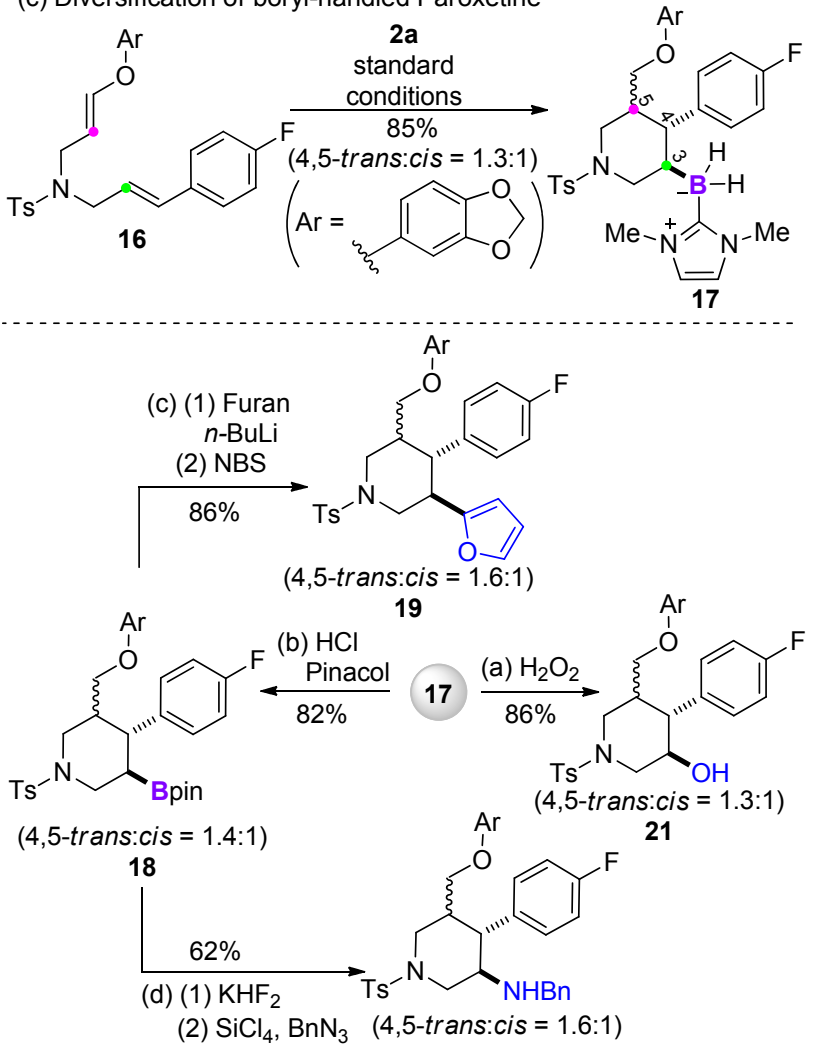

20

图式 3 1,6-二烯的自由基硼化环化反应

Scheme 3 Radical borylative cyclization of 1,6-dienes

合作者 ${ }^{[25]}$ 利用嗍自由基和非末端炔烃发生加成反应, 实现了反式烯基硼化合物的构建. 此反应的 $Z / E$ 选择性 同样与经典的硼氢化反应相反.

$\alpha$-羰基硼化合物在合成化学中具有重要的应用价 值 ${ }^{[26]}$. 然而, 目前已知的 $\alpha$-羰基硼化合物的合成方法往 往需要多步反应且较为耗时 ${ }^{[27]}$. 因此, 开发便捷的 $\alpha$-羰 基嗍化合物的合成方法具有重要意义. 2019 年, 我们课 (a) Synthesis of boron-handled amidines
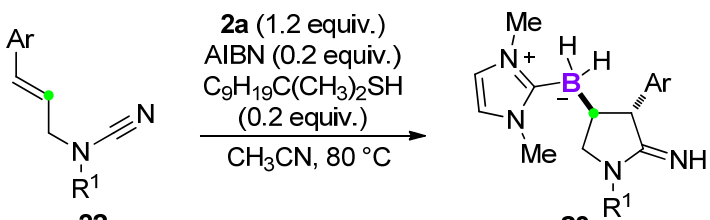

22

$23^{\mathrm{R}^{1}}$

$\mathrm{R}^{1}=\mathrm{Ts}, \mathrm{Ar}, \mathrm{Bn}, \mathrm{Boc}$

18 examples, $51 \% \sim 91 \%$

(b) Synthesis of boron-handled quinazolinones

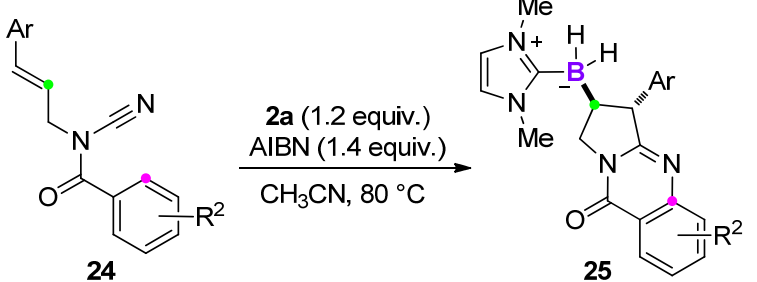

24

25

$3 \%$

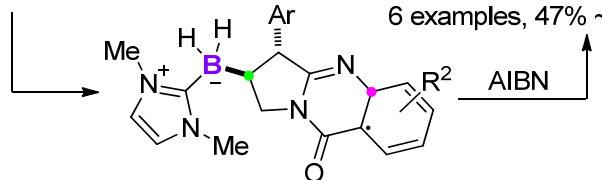

图式 4 硼官能团取代的环脒和喹唑啉酮的合成

Scheme 4 Synthesis of boron-handled amidines and quinazolinones

题组 ${ }^{[28]}$ 利用廉价易得的 $\alpha, \beta$-不饱和羰基化合物和氮杂 环卡宾一嗍烷为原料，实现了 $\alpha$-羰基嗍化合物 27 的高效 合成(Scheme 5a). 该反应表现出特异的非常规 $\alpha$-区域选 择性. 我们通过密度泛函理论(DFT)计算和动力学研究, 探究了该自由基硼氢化反应 $\alpha$-区域选择性的成因. 结果 表明，对于 $\beta$-芳基- $\alpha, \beta$-不饱和羰基化合物的硼氢化反 应，其 $\alpha$-区域选择性是由氮杂环卡宾-硼自由基加成步 决定，氮杂环卡宾-嗍自由基加成于羰基的 $\alpha$ 位在热力

(a) Radical hydroboration of $\alpha, \beta$-unsaturated compounds 2a (1.0 equiv.)

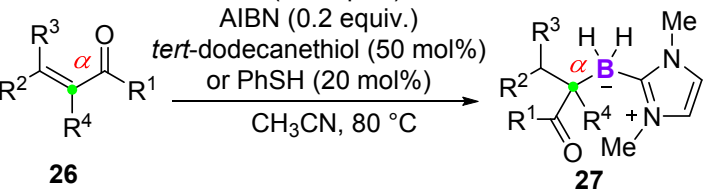

1.2 equiv. $\quad 34$ examples, $39 \% \sim 95 \%$

(b) Synthesis of boron-hadled benzazocines (5 examples)

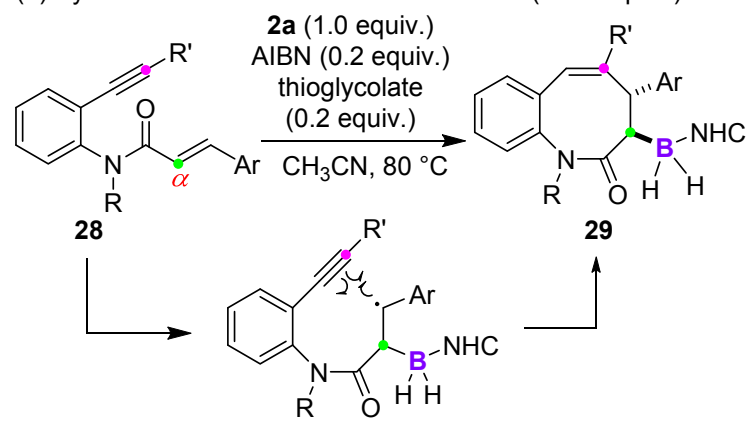

图式 $5 \alpha, \beta$-不饱和羰基化合物的自由基硼氢化反应

Scheme 5 Radical hydroboration of $\alpha, \beta$-unsaturated compounds 
学上更加有利. 对于 $\beta$-烷基- $\alpha, \beta$-不饱和羰基化合物的硼 氢化反应, $\alpha$-区域选择性是由氢转移步决定, $\alpha$ 加成的氢 转移步所需的活化能更低. 在此工作中, 我们还开发了 一种氮杂环卡宾-嗍烷取代的苯并八元内酰胺的合成方 法(Scheme $5 b)^{[29]}$. 利用制得 $\alpha$-羰基硼化合物 27 和氮杂 环卡宾一硼烷取代的苯并八元内酰胺 $\mathbf{2 9}$ 为原料, 实现了 多种后续衍生化和转化.

2020 年, 朱成建、谢劲和黎书华课题组 ${ }^{[30]}$ 利用氮杂 环卡宾-硼烷和 $\alpha, \beta$-不饱和酰胺为原料, 在光催化条件 下，以优良的 $\beta$-区域选择性实现了 $\beta$-羰基嗍化合物的构 建.

在上述结果的基础上, 为进一步加深对硼自由基与 碳碳双键反应性能的理解, 我们 ${ }^{[31]}$ 又研究了硼自由基 与其他贫电子烯烃的硼氢化反应. 结果表明, 该硼自由 基仍可以选择性加成到缺电子烯烃的 $\alpha$ 位, 得到多种类 型的 $\alpha$-硼代化合物, 如 $\alpha$-硼代腈、三氟甲基、磷酸酯、 砜类化合物以及偕二硼化合物 31 (Scheme 6).

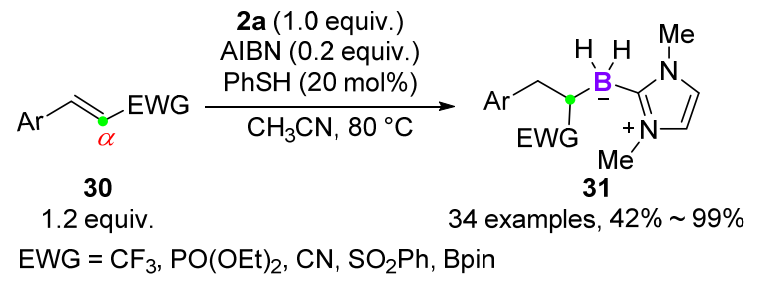

图式 6 贫电子烯烃的自由基嗍氢化反应

Scheme 6 Radical hydroboration of electron-poor alkenes

有机氟化合物具有独特的理化性能, 如优良的物理 和化学稳定性, 能够增强药物分子代谢的稳定性等, 被 广泛的应用于合成化学、药物化学和材料科学等领 域 ${ }^{[32]}$. 因此, 发展高效的方法来合成有机氟化合物具有 重要意义 ${ }^{[33]}$. 由于 $\mathrm{C}-\mathrm{B}$ 键易于转化的特性, 合成磞官 能团取代的有机氟化合物易于实现母体结构的多样性 转化和修饰, 能够为结构多样的有机氟化合物的合成提 供一种简单、易行的方法. 2019 年, 我们课题组 ${ }^{[34]}$ 使用 芳基和羰基取代偕二氟烯烃为原料, 通过自由基硼氢化 反应, 实现了 $\alpha$-二氟烷基硼化合物 33 的制备(Scheme 7a). 通过 DFT 计算, 发现此反应专一的 $\alpha$-区域选择性 是由氮杂环卡宾-硼自由基加成步所决定. 在热力学和 动力学上, 氮杂环卡宾-嗍自由基加成在氟原子的 $\alpha$ 位 都有利. 单氟烯烃是酰胺的等电子体, 在生物活性分子 中较为常见 ${ }^{[35]}$. 使用 $\mathrm{KO}^{t} \mathrm{Bu}$ 对 $\alpha$-二氟烷基嗍化合物 33a 进行处理, 反应能够以定量的收率得到氮杂环卡宾-硼 烷取代的单氟烯烃 34 (Scheme 7b). 不经提纯, 氮杂环 卡宾一嗍烷取代的单氟烯烃粗产品能够直接用作亲核性 的烯基化试剂和多种亲电试剂发生偶联反应, 实现了多 种单氟烯烃的高效合成. (a) Radical hydroboration of gem-difluoroalkenes

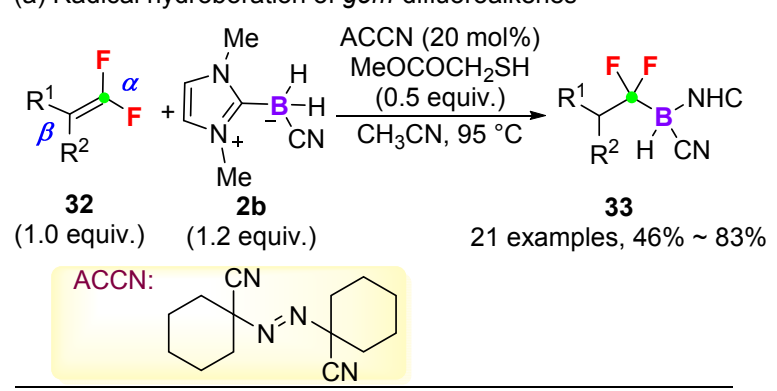

(b) Transformations of monofluoroalkenyl borons
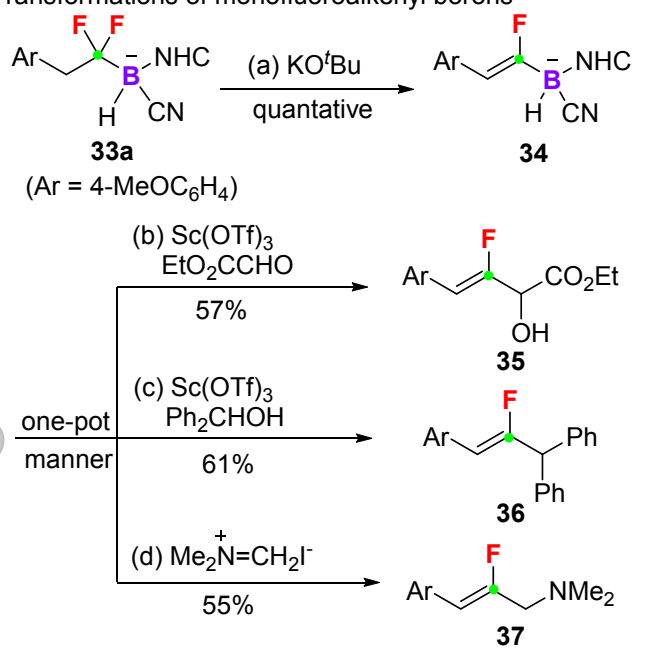

图式 7 偕二氟烯烃的自由基嗍氢化反应

Scheme 7 Radical hydroboration of gem-difluoroalkenes

在我们开展这项研究工作时, 王洪根课题组 ${ }^{[36]}$ 也 实现了一例类似的偕二氟烯烃的嗍氢化反应，反应同样 以优良的区域选择性实现了 $\alpha$-二氟烷基硼化合物的合 成.

\section{3 光催化的自由基硼氢化反应}

尽管使用氮杂环卡宾-硼烷作为硼自由基前体能够 实现多种有机嗍化合物的高效构建，但氮杂环卡宾一嗍 自由基的产生方式却非常单一，主要局限于自由基引发 剂㩲取氮杂环卡宾一嗍烷的氢原子. 这种单一的引发方 式也在一定程度上限制了自由基硼化反应的反应类型. 因此，发展硼自由基新的产生方式，发现新的反应模式 能为有机硼化合物多样性合成提供诸多可能性. 2020 年, 我们课题组 ${ }^{\left[{ }^{37]}\right.}$ 开发了一种光催化、单电子氧化产生 硼自由基的新方式(Scheme 8). 在此工作中, 氮杂环卡 宾-嗍自由基 I 通过单电子氧化产生(Scheme 8a). 随后, 硼自由基 I 和反应中原位生成的自由基负离子 II 发生自 由基偶联得到中间体 III. 中间体 III 离去氟负离子得到 脱氟硼化产物 39. 此外, 我们还利用芳基氰化物作为芳 基化试剂，在光照条件下，实现了烯烃的芳嗍化反应 (Scheme $8 b$ ). 在该反应中, 氧化生成的嗍自由基 $\mathbf{I}$ 加成 于烯烃上得到相应的苄基自由基中间体 IV. 中间体 IV 
(a) Defluoroborylation of trifluoromethyl-substituted alkenes
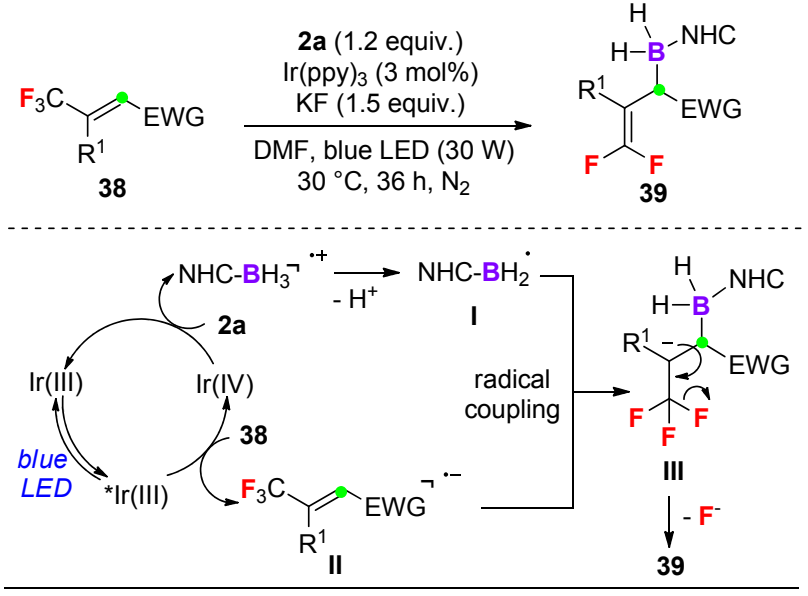

(b) Radical arylboration of alkenes
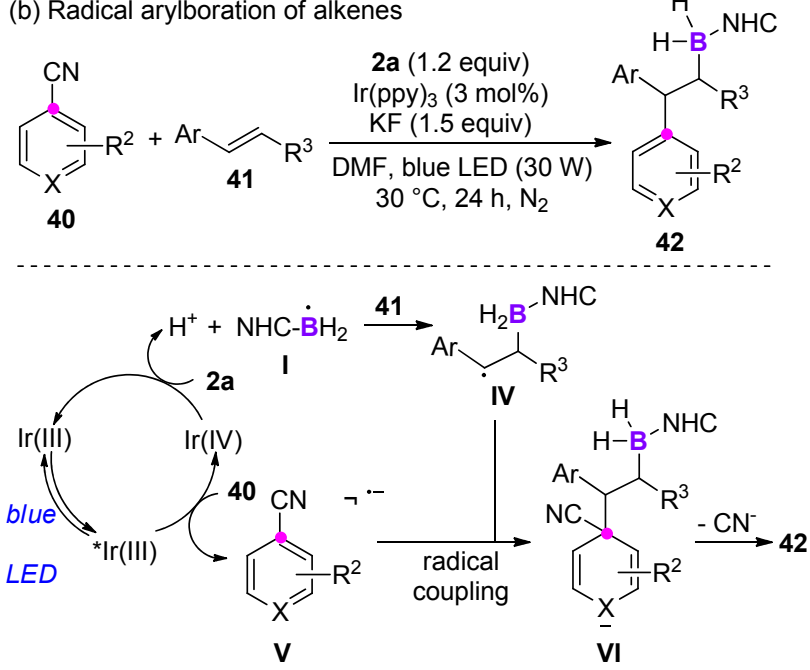

图式 8 光催化的硼化反应

Scheme 8 Borylation reaction enabled by photoredox catalysis

和原位生成的芳基氰化物的自由基负离子 $\mathbf{V}$ 发生自由 基偶联, 得到相应的中间体 VI. 中间体 VI 离去氰基负 离子得到最终的芳嗍化产物 $\mathbf{4 2}$. 该光催化、单电子氧化 产生嗍自由基的策略为多官能团取代的有机嗍化合物 的合成提供了一种新的思路和方法. 我们通过循环伏 安法、荧光淬灭实验、自由基钟反应等手段，证实了氮 杂环卡宾一嗍烷经由氧化产生氮杂环卡宾-硼自由基的 机理.

与此同时, 阳华课题组 ${ }^{[38]}$ 利用类似的光催化反应 策略，实现了一例嗍自由基促进的脱氟硼化反应和自由 基嗍氢化反应. 此外, Curran 课题组 ${ }^{[39]}$ 也利用此单电子 氧化生成硼自由基的策略, 实现了一例贫电子芳环的 1,4-嗍氢化反应, 为硼官能团取代的 1,4-环已二烯的合 成提供了一种可行的方法.

\section{2 嗍自由基催化反应研究进展}

催化反应作为高效构建有机分子的一种重要途径
和方法，被广泛用于化合物的合成，其中过渡金属催 化、有机小分子催化以及酶催化已被广泛研究. 然而, 利用自由基物种作为催化剂的反应却鲜有报道. 这可能 是因为自由基物种一般活性较高，很难实现有效的催化 循环. 基于自由基具有独特的化学活性，如果能开发高 效的催化反应, 将有望实现其他方法难以实现的有机转 化和合成. 目前, 自由基催化的反应主要局限于硫自由 基和锡自由基催化的烯基环丙烷和烯烃的环化反应 ${ }^{[40]}$. 2014 年, Maruoka 课题组 ${ }^{[41]}$ 报道了一例手性硫自由基催 化的不对称合成反应. 2019 年, 肖文精和陈加荣课题 组 ${ }^{[2]}$ 利用烯烃和烯丙基砜为原料, 氮自由基作为催化 剂，实现了一例烯烃的分子间双官能团化反应，开辟了 氮自由基在催化领域的新应用. 2019 年，我们课题组 ${ }^{[43]}$ 利用氮杂环卡宾一嗍自由基作为催化剂, 实现了一例有 机分子骨架的重排反应(Scheme 9). 在此反应中，硼自 由基首先加成于芳环共轭的碳碳参键上，得到相应的烯 基自由基中间体 I (Scheme 9b). 随后，发生两次芳基迁 移得到自由基中间体 VI. 自由基中间体 VI 发生 $\beta$-断裂 得到喹啉酮产物 44, 并重新生成氮杂环卡宾-硼自由基, 实现了反应的催化循环. 为了证实此机理的合理性，我 们对此反应的反应历程进行了 DFT 计算, 其结果完全 支持此嗍自由基催化的反应机理. 该工作开辟了嗍自由 基在自由基催化领域的新应用，为后续氮杂环卡宾-硼 自由基催化方面工作的开展奠定了坚实的基础.

\section{3 嗍自由基促进的还原反应研究进展}

自由基还原反应在有机合成中被广泛应用. 其中, 利用 ${ }^{n} \mathrm{Bu}_{3} \mathrm{Sn}$ •能够高效地实现多种自由基还原反应，如 还原去卤、还原脱氧反应等 ${ }^{[4]}$. 然而，由于有机锡试剂 具有较强的毒性，极大地限制了其在有机合成中的应 用 ${ }^{[45]}$. 近年来, 多种不同的路易斯碱一硼烷作为 ${ }^{n} \mathrm{Bu}_{3} \mathrm{Sn}-$ $\mathrm{H}$ 的替代品已经被开发和利用，实现了多种不同的自由 基还原反应. 例如, Curran 课题组 ${ }^{[3-4]}$ 利用氮杂环卡宾嗍自由基实现了一系列有机卤代物，黄原酸酯 ${ }^{[2]}$ 和有机 㲵化物 ${ }^{[5]}$ 的还原反应.

硫代酰胺廉价易得、来源广泛，在有机合成中具有 重要的应用价值 ${ }^{[46]}$. 例如, 还原硫代酰胺能够得到有机 胺产物. 经典的硫代酰胺的还原方法包括: Raney 镍氢 化还原 ${ }^{[46]}, \mathrm{Zn} / \mathrm{HCl}$ 还原 ${ }^{[47]}$ 等. 然而, 这些经典的还原方 法具有反应条件苛刻，官能团容忍性差，操作复杂等缺 点. 因此, 发展一种条件温和、操作简便的硫代酰胺的 还原方法具有重要意义. 2018 年, 我们课题组 ${ }^{[48]}$ 利用对 二甲胺基吡啶-嗍自由基( $\mathrm{DMAP}-\mathrm{BH}_{2} \bullet$ ), 在温和的条件 下实现了硫代酰胺的还原反应(Scheme 10a). 在此反应 中, 对二甲胺基吡啶一嗍自由基能够选择性地加成于硫 
(a) NHC-boryl radical catalysis for skeletal rearrangement

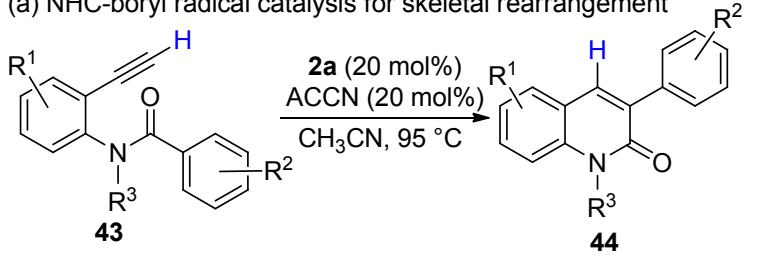

20 examples, $21 \% \sim 97 \%$

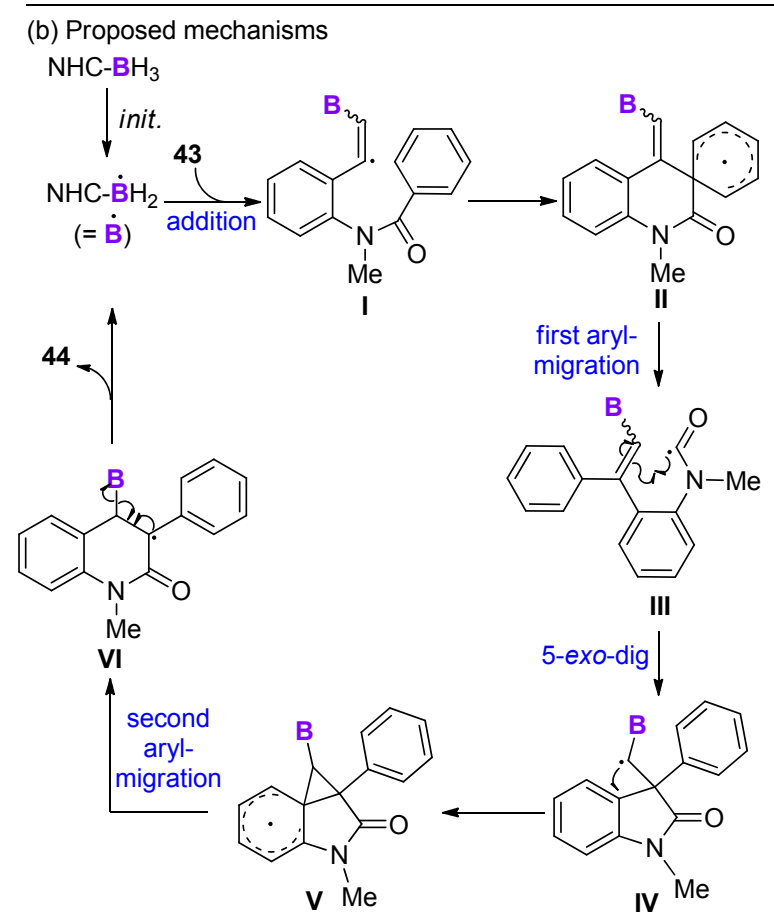

图式 9 嗍自由基促进的重排反应

Scheme 9 NHC-boryl radical catalysis for skeletal rearrangement

代酰胺的硫原子上, 得到 $\alpha$-硫胺烷基自由基中间体 $\mathbf{I}$. 随后, 自由基中间体 $\mathbf{I}$ 从苯硫酚篗取氢原子得到中间体 II 和硫自由基. 中间体 II 离去 DMAP- $\mathrm{BH}_{2} \mathrm{SH}$ 副产物生 成相应的亚胺中间体. 在过量的对二甲胺基吡啶-硼烷 还原作用下，亚胺中间体被还原生成相应的有机胺产物 46. 反应中生成的硫自由基能够进一步极性匹配的算取 对二甲胺基吡定一硼烷( $\left.\mathrm{DMAP}-\mathrm{BH}_{3}\right)$ 上的氢原子重新生 成对二甲胺基吡啶一嗍自由基和苯硫酚, 实现了此还原 反应的催化循环. 然而, 此还原体系对烷基硫代酰胺并 不适用, 原料基本上完全回收. 当使用含有分子内碳碳 双键的硫代酰胺为原料进行反应时, 反应生成的 $\alpha$-硫胺 烷基自由基中间体能够被分子内碳碳双键所捕获, 实现 多种氮杂环化合物的高效构建 (Scheme 10c) ${ }^{[49]}$.

在自由基反应中, 碳自由基是一类重要的中间体. 因此, 多种产生碳自由基的方法已经被开发和利用. 羧 酸化合物具有来源广泛, 廉价易得等优点. 利用羧酸脱 羧, 尤其是通过邻苯二甲酰亚胺羧酸酯(NHPI 酯)脱羧, 是产生碳自由基的重要方式之一 ${ }^{[50]}$. 基于傅尧课题组 NHPI 酯脱羧的先驱工作 ${ }^{[51]}$, 近年来, 多种 NHPI 酯脱羧 (a) Desulfurizative reduction reactions

$$
\text { TBHN: }
$$

(b) Proposed mechanism

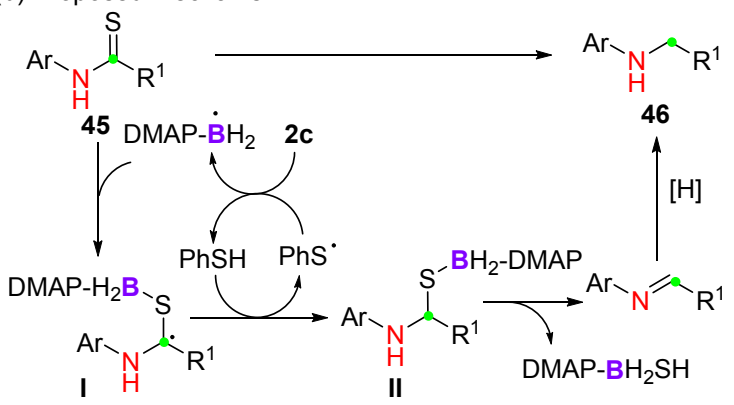

(c) Radical annulation of thioamides (8 examples)

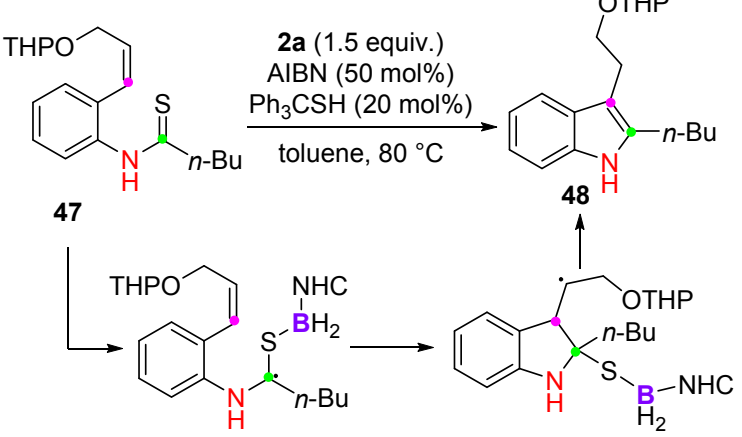

图式 10 硫代酰胺的脱硫还原和环化反应

Scheme 10 Desulfurizative reduction and annulation of thioamides

官能团化反应已被广泛开发和利用. 然而, NHPI 酯脱羧 主要依赖于贵重的过渡金属催化和光催化两种方式. 因 此, 发展一种不添加过渡金属的 NHPI 酯脱羧的方法具 有重要意义. 在硫代酰胺还原工作的基础上, 2019 年, 我们课题组 ${ }^{[52]}$ 开发了一例路易斯碱一硼自由基促进的 NHPI 酯的 Barton 脱羧反应和 Giese 反应(Scheme 11). 在 此反应中，对二甲胺基吡啶一嗍自由基能够选择性地加 成于 NHPI 酯的羰基氧原子上得到相应的 $\alpha$-氮氧烷基自 由基中间体 I (Scheme 11c) ${ }^{[53]}$. 随后，中间体 $\mathbf{I}$ 发生 $\beta$-断 裂，释放出 $\mathrm{CO}_{2}$ ，生成亲核性的烷基自由基中间体 II. 在脱羧 Giese 反应中, 亲核性的碳自由基中间体 II 加成 于 $\alpha, \beta$-不饱和羰基化合物得到亲电性的 $\alpha$-羰基碳自由基 中间体. $\alpha$-羰基碳自由基进一步极性匹配地从对二甲胺

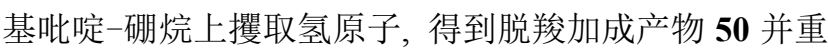
新生成对二甲胺基吡啶一硼自由基，实现了脱羧 Giese 反 应的催化循环. 在 Barton 脱羧反应中, 亲核性的烷基自 由基中间体 II 能够极性匹配地篗取苯硫酚上的氢原子, 
(a) Giese reaction of NHPI esters

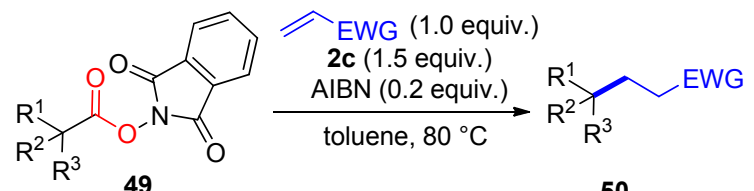

$1^{\circ}, 2^{\circ}, 3^{\circ}$ alkyl acid 17 examples, $51 \% \sim 85 \%$

(b) Barton decarborxylation of NHPI esters

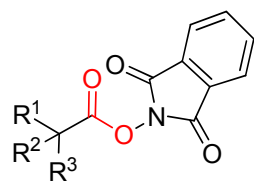

51

$1^{\circ}, 2^{\circ}, 3^{\circ}$ alkyl acid

c) Proposed mechanism 2c (1.1 equiv.) AIBN ( 0.2 equiv. $)$ $\stackrel{\mathrm{PhSH}(0.2 \text { equiv. })}{\longrightarrow} \mathrm{R}^{2} \mathrm{R}^{1}{ }^{\mathrm{H}}$

52 8 examples, $57 \% \sim 86 \%$

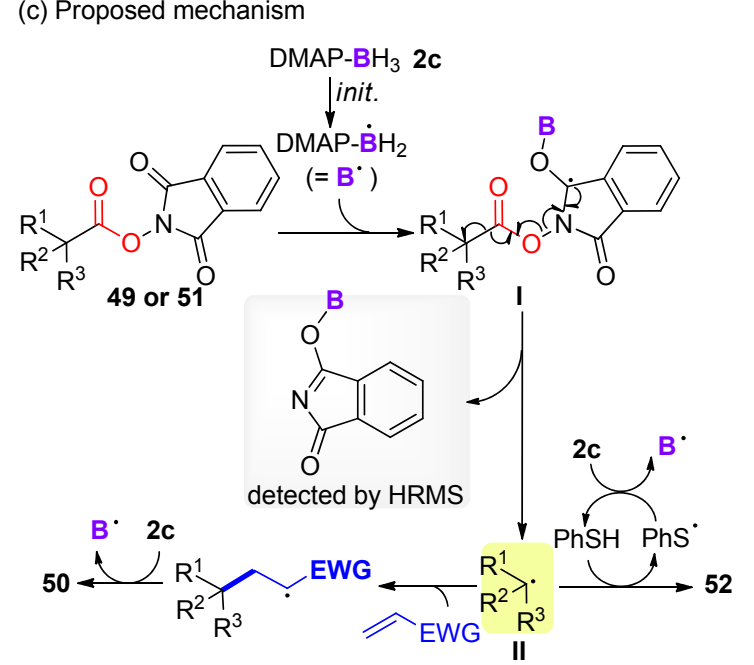

图式 11 NHPI 酯的 Barton 脱羧反应和 Giese 反应

Scheme 11 Barton decarborxylation and Giese reaction of NHPI esters

生成相应的脱羧还原产物 52 和硫自由基. 硫自由基进 一步极性匹配地从对二甲胺基吡啶－嗍烷䣮取氢原子， 重新生成对二甲胺基吡啶一磞自由基和苯硫酚，实现了 脱羧还原反应的催化循环.

\section{4 结论与展望}

近年来，我们课题组利用路易斯碱-嗍自由基实现 了一系列有用的合成反应, 能够实际解决有机合成中存 在的一些问题和难题. 这些反应具有条件温和, 官能团 容忍性好, 产率高, 化学、区域选择性优良等优点. 硼自 由基物种的结构和反应性能相较于其他主族元素自由 基物种具有一定差异性, 为新型合成反应的发现和发展 提供了诸多可能.

尽管利用路易斯碱一硼自由基已经能够实现多样性 的合成反应，但是对于路易斯碱一硼自由基的应用仍存 在一些问题和挑战. 例如, 路易斯碱-硼烷具有较强的 还原性; 反应类型不够多样化; 如何实现不对称催化等.
因此，致力于新型硼自由基前体的设计和探索，进一步 深入探究路易斯碱-硼自由基新的反应性能等具有重要 意义.

\section{作者简介}

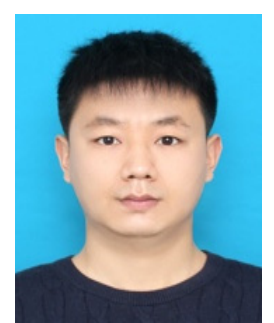

靳继康, 2014 年毕业于新乡学院化学与化工学院, 同年进入中国科学技术大学化学与材料科学学院有机 化学专业攻读博士学位, 师从汪义丰教授, 2020 年获得 博士学位. 主要从事嗍自由基促进的合成反应研究.

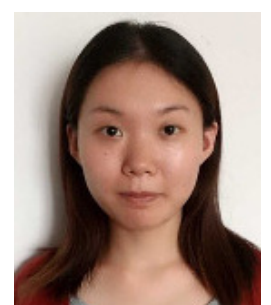

夏慧敏, 2015 年毕业于山东师范大学化学院，同年 进入中国科学技术大学化学与材料科学学院有机化学 专业攻读博士学位, 师从汪义丰教授. 主要从事有机合 成方法学的研究.

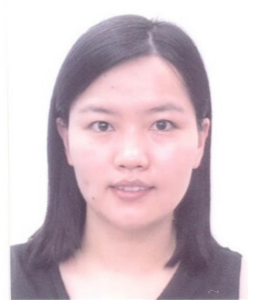

张风莲, 博士. 2015 年毕业于新加坡南洋理工大学, 获得理学博士学位, 师从 Shunsuke Chiba 教授. 2016 年 至 2019 年, 在中国科学技术大学汪义丰教授课题组从 事博士后研究. 2019 年转聘为特任副研究员. 主要从事 有机嗍化学以及相关机理研究.

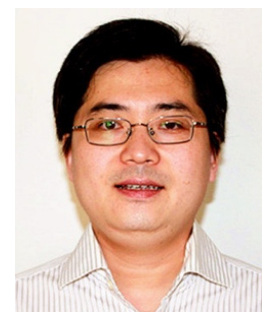

汪义丰，教授. 2003 年本科毕业于华中师范大学, 
2006 年于南开大学获硕士学位, 师从杨华铮教授. 2011 年于新加坡南洋理工大学获博士学位, 师从 Koichi Narasaka 和 Shunsuke Chiba 教授. 2011 2015 年继续在南 洋理工大学从事博士后研究, 并于 $2012 \sim 2014$ 年获 “Lee Kuan Yew Postdoctoral Fellowship” 资助. 2015 年 9 月至今任中国科学技术大学教授. 主要从事有机合成方 法学、嗍自由基化学和具有生物活性物质的合成等方面 的研究.

\section{References}

[1] Roberts, B. P. Chem. Soc. Rev. 1999, 28, 25.

[2] (a) Ueng, S.-H.; Makhlouf Brahmi, M.; Derat, É.; Fensterbank, L.; Lacôte, E.; Malacria, M.; Curran, D. P. J. Am. Chem. Soc. 2008, 130, 10082.

(b) Ueng, S.-H.; Fensterbank, L.; Lacôte, E.; Malacria, M.; Curran, D. P. Org. Lett. 2010, 12, 3002.

[3] Pan, X.; Lacôte, E.; Lalevée, J.; Curran, D. P. J. Am. Chem. Soc. 2012, 134, 5669.

[4] (a) Pan, X.; Lalevée, J.; Lacôte, E.; Curran, D. P. Adv. Synth. Catal. 2013, 355, 3522.

(b) Ueng, S.-H.; Fensterbank, L.; Lacôte, E.; Malacria, M.; Curran, D. P. Org. Biomol. Chem. 2011, 9, 3415.

[5] Kawamoto, T.; Geib, S. J.; Curran, D. P. J. Am. Chem. Soc. 2015, 137, 8617.

[6] Tehfe, M.-A.; Makhlouf Brahmi, M.; Fouassier, J.-P.; Curran, D. P.; Malacria, M.; Fensterbank, L.; Lacôte, E.; Lalevée, J. Macromolecules 2010, 43, 2261.

[7] Hall, D. G. Boronic Acids: Preparation and Applications in Organic Synthesis, Medicine and Materials, 2nd ed., Wiley-VCH, Weinheim, Germany, 2011.

[8] (a) Burgess, K.; Ohlmeyer, M. J. Chem. Rev. 1991, 91, 1179.

(b) Brown, H. C.; Singaram, B. Acc. Chem. Res. 1988, 21, 287.

(c) Brown, H. C.; Rao, B. C. S. J. Am. Chem. Soc. 1956, 78, 5694.

[9] (a) Ishiyama, T.; Murata, M.; Miyaura, N. J. Org. Chem. 1995, 60, 7508 .

(b) Ishiyama, T.; Matsuda, N.; Miyaura, N.; Suzuki, A. J. Am. Chem. Soc. 1993, 115, 11018.

[10] (a) Wille, U. Chem. Rev. 2013, 113, 813.

(b) Li, C.; Zhu, C. Acta Chim. Sinica 2019, 77, 771 (in Chinese). (李超忠, 朱晨, 化学学报, 2019, 77, 771.)

[11] (a) Friese, F. W.; Studer, A. Chem. Sci. 2019, 10, 8503. (b) Taniguchi, T. Eur. J. Org. Chem. 2019, $2019,6308$.

(c) Zhang, F.-L.; Wang, Y.-F. Reactions through Radical Boryl Moieties in Science of Synthesis: Advances in Organoboron Chemistry towards Organic Synthesis, Eds.: Fernnádez, G., Thieme Verlag KG, Stuttgart, Germany, 2019, pp. 355 392.

(d) Yang, J.-M.; Li, Z.-Q.; Zhu, S.-F. Chin. J. Org. Chem. 2017, 37, 2481 (in Chinese).

(杨吉民，李子奇，朱守非，有机化学, 2017, 37, 2481.)

(e) Curran, D. P.; Solovyev, A.; Makhlouf Brahmi, M.; Fensterbank, L.; Malacria, M.; Lacôte, E. Angew. Chem., Int. Ed. 2011, 50, 10294.

[12] Ren, S.-C.; Zhang, F.-L.; Qi, J.; Huang, Y.-S.; Xu, A.-Q.; Yan, H.-Y.; Wang, Y.-F. J. Am. Chem. Soc. 2017, 139, 6050.

[13] (a) Walton, J. C.; Brahmi, M. M.; Fensterbank, L.; Lacôte, E.; Malacria, M.; Chu, Q.; Ueng, S.-H.; Solovyev, A.; Curran, D. P. J. Am. Chem. Soc. 2010, 132, 2350.

(b) Ueng, S.-H.; Solovyev, A.; Yuan, X.; Geib, S. J.; Fensterbank, L.; Lacôte, E.; Malacria, M.; Newcomb, M.; Walton, J. C.; Curran, D. P. J. Am. Chem. Soc. 2009, 131, 11256.

[14] Solovyev, A.; Chu, Q.; Geib, S. J.; Fensterbank, L.; Malacria, M.; Lacôte, E.; Curran, D. P. J. Am. Chem. Soc. 2010, 132, 15072.

[15] Nerkar, S.; Curran, D. P. Org. Lett. 2015, 17, 3394.
[16] Watanabe, T.; Hirose, D.; Curran, D. P.; Taniguchi, T. Chem.-Eur. J. 2017, 23, 5404.

[17] (a) Taylor, R. D.; MacCoss, M.; Lawson, A. D. G. J. Med. Chem. 2014, 57, 5845 .

(b) Luca, C.; Daniela, B. Curr. Med. Chem. 2006, 13, 65.

[18] (a) Zheng, Y.; Tice, C. M.; Singh, S. B. Bioorg. Med. Chem. Lett. 2014, 24, 3673.

(b) Aldeghi, M.; Malhotra, S.; Selwood, D. L.; Chan, A. W. E. Chem. Biol. Drug Des. 2014, 83, 450.

[19] (a) Kubota, K.; Watanabe, Y.; Hayama, K.; Ito, H. J. Am. Chem. Soc. 2016, 138, 4338.

(b) Yamamoto, E.; Takenouchi, Y.; Ozaki, T.; Miya, T.; Ito, H. J. Am. Chem. Soc. 2014, 136, 16515.

(c) Sasaki, Y.; Zhong, C.; Sawamura, M.; Ito, H. J. Am. Chem. Soc. 2010, 132, 1226.

(d) Lee, K.-s.; Zhugralin, A. R.; Hoveyda, A. H. J. Am. Chem. Soc. 2009, 131, 7253 .

(e) Bonet, A.; Gulyás, H.; Fernández, E. Angew. Chem., Int. Ed. 2010, 49, 5130.

(f) Feng, X.; Yun, J. Chem. Commun. 2009, 6577.

(g) Bonet, A.; Sole, C.; Gulyás, H.; Fernández, E. Chem. Asian J. 2011, 6, 1011 .

[20] Qi, J.; Zhang, F.-L.; Huang, Y.-S.; Xu, A.-Q.; Ren, S.-C.; Yi, Z.-Y.; Wang, Y.-F. Org. Lett. 2018, 20, 2360.

[21] (a) Thomas, G. L.; Johannes, C. W. Curr. Opin. Chem. Biol. 2011, 15,516 .

(b) Welsch, M. E.; Snyder, S. A.; Stockwell, B. R. Curr. Opin. Chem. Biol. 2010, 14, 347.

(c) Oehlrich, D.; Prokopcova, H.; Gijsen, H. J. M. Bioorg. Med. Chem. Lett. 2014, 24, 2033.

(d) Edwards, P. D.; Albert, J. S.; Sylvester, M.; Aharony, D.; Andisik, D.; Callaghan, O.; Campbell, J. B.; Carr, R. A.; Chessari, G.; Congreve, M.; Frederickson, M.; Folmer, R. H. A.; Geschwindner, S.; Koether, G.; Kolmodin, K.; Krumrine, J.; Mauger, R. C.; Murray, C. W.; Olsson, L.-L.; Patel, S.; Spear, N.; Tian, G. J. Med. Chem. 2007, 50, 5912.

(e) Shankaran, K.; Donnelly, K. L.; Shah, S. K.; Guthikonda, R. N.; MacCoss, M.; Humes, J. L.; Pacholok, S. G.; Grant, S. K.; Kelly, T. M.; Wong, K. K. Bioorg. Med. Chem. Lett. 2004, 14, 4539.

(f) Kshirsagar, U. A. Org. Biomol. Chem. 2015, 13, 9336.

[22] Jin, J.-K.; Zhang, F.-L.; Zhao, Q.; Lu, J.-A.; Wang, Y.-F. Org. Lett. 2018, 20, 7558.

[23] (a) Demay, S.; Volant, F.; Knochel, P. Angew. Chem., Int. Ed. 2001, 40, 1235.

(b) Evans, D. A.; Fu, G. C.; Hoveyda, A. H. J. Am. Chem. Soc. 1992, 114, 6671.

[24] Zhou, N.; Yuan, X.-A.; Zhao, Y., Xie, J.; Zhu, C. Angew. Chem., Int. Ed. 2018, 57, 3990.

[25] Shimoi, M.; Watanabe, T.; Maeda, K.; Curran, D. P.; Taniguchi, T. Angew. Chem., Int. Ed. 2018, 57, 9485.

[26] (a) Brauer, D. J.; Bürger, H.; Buchheim-Spiegel, S.; Pawelke, G. Eur. J. Inorg. Chem. 1999, 1999, 255.

(b) Bai, J.; Burke, L. D.; Shea, K. J. J. Am. Chem. Soc. 2007, 129, 4981.

(c) Caskey, S. R.; Stewart, M. H.; Johnson, M. J. A.; Kampf, J. W. Angew. Chem., Int. Ed. 2006, 45, 7422.

(d) Bell, N. J.; Cox, A. J.; Cameron, N. R.; Evans, J. S. O.; Marder, T. B.; Duin, M. A.; Elsevier, C. J.; Baucherel, X.; Tulloch, A. A. D.; Tooze, R. P. Chem. Commun. 2004, 1854.

(e) Ansorge, A.; Brauer, D. J.; Bürger, H.; Hagen, T.; Pawelke, G. Angew. Chem., Int. Ed. 1993, 32, 384.

(f) Denis, St. J. D.; He, Z.; Yudin, A. K. ACS Catal. 2015, 5, 5373.

(g) He, Z.; Zajdlik, A.; Yudin, A. K. Acc. Chem. Res. 2014, 47, 1029.

[27] (a) Kan, S. B. J.; Huang, X.; Gumulya, Y.; Chen, K.; Arnold, F. H. Nature 2017, 552, 132.

(b) Yang, J.-M.; Zhao, Y.-T.; Li, Z.-Q.; Gu, X.-S.; Zhu, S.-F.; Zhou, Q.-L. ACS Catal. 2018, 8, 7351. 
(c) Cheng, Q.-Q.; Zhu, S.-F.; Zhang, Y.-Z.; Xie, X.-L.; Zhou, Q.-L. J. Am. Chem. Soc. 2013, 135, 14094.

(d) Allen, T. H.; Kawamoto, T.; Gardner, S.; Geib, S. J.; Curran, D. P. Org. Lett. 2017, 19, 3680

(e) Li, X.; Curran, D. P. J. Am. Chem. Soc. 2013, 135, 12076.

(f) Corless, V. B.; Holownia, A.; Foy, H.; Mendoza-Sanchez, R.; Adachi, S.; Dudding, T.; Yudin, A. K. Org. Lett. 2018, 20, 5300.

(g) Lv, W.-X.; Zeng, Y.-F.; Li, Q.; Chen, Y.; Tan, D.-H.; Yang, L.; Wang, H. Angew. Chem., Int. Ed. 2016, 55, 10069.

(h) Li, J.; Burke, M. D. J. Am. Chem. Soc. 2011, 133, 13774.

(i) He, Z.; Yudin, A. K. J. Am. Chem. Soc. 2011, 133, 13770.

[28] Ren, S.-C.; Zhang, F.-L.; Xu, A.-Q.; Yang, Y.; Zheng, M.; Zhou, X.; Fu, Y.; Wang, Y.-F. Nat. Commun. 2019, 10, 1934.

[29] Liu, L.; Chen, Q.; Wu, Y.-D.; Li, C. J. Org. Chem. 2005, 70, 1539.

[30] Zhu, C.; Dong, J.; Liu, X.; Gao, L.; Zhao, Y.; Xie, J.; Li, S.; Zhu, C. Angew. Chem., Int. Ed. 2020, 59, 12817.

[31] Huang, Y.-S.; Wang, J.; Zheng, W.-X.; Zhang, F.-L.; Yu, Y.-J.; Zheng, M.; Zhou, X.; Wang, Y.-F. Chem. Commun. 2019, 55, 11904.

[32] (a) Purser, S.; Moore, P. R.; Swallow, S.; Gouverneur, V. Chem Soc. Rev. 2008, 37, 320.

(b) Hagmann, W. K. J. Med. Chem., 2008, 51, 4359.

(c) Babudri, F.; Farinola, G. M.; Naso, F.; Ragni, R. Chem. Commun. 2007, 1003 .

(d) O’Hagan, D.; S. Rzepa, H.; Chem. Commun. 1997, 645.

[33] Hiyama, T.; Yamamoto, H. In Organofluorine Compounds: Chemistry and Applications, Eds.: Hiyama T.; Yamamoto, H., Springer Berlin Heidelberg, Berlin, 2000, pp. 25 76.

[34] Jin, J.-K.; Zheng, W.-X.; Xia, H.-M.; Zhang, F.-L.; Wang, Y.-F. Org. Lett. 2019, 21, 8414

[35] (a) Couve-Bonnaire, S.; Cahard, D.; Pannecoucke, X. Org. Biomol. Chem. 2007, 5, 1151 .

(b) Vedejs, E.; Fields, S. C.; Hayashi, R.; Hitchcock, S. R.; Powell, D. R.; Schrimpf, M. R. J. Am. Chem. Soc. 1999, 121, 2460

(c) Daubresse, N.; Chupeau, Y.; Francesch, C.; Lapierre, C.; Pollet, B.; Rolando, C. Chem. Commun. 1997, 1489.

(d) Van der Veken, P.; Senten, K.; Kertèsz, I.; De Meester, I.; Lambeir, A.-M.; Maes, M.-B.; Scharpé, S.; Haemers, A.; Augustyns, K. J. Med. Chem. 2005, 48, 1768 .

[36] Liu, X.; Lin, E.-E.; Chen, G.; Li, J.-L.; Liu, P.; Wang, H. Org. Lett. 2019, 21,8454 .

[37] Qi, J.; Zhang, F.-L.; Jin, J.-K.; Zhao, Q.; Li, B.; Liu, L.-X.; Wang, Y.-F. Angew. Chem., Int. Ed. 2020, 59, 12876.

[38] Xia, P.-J.; Song, D.; Ye, Z.-P.; Hu, Y.-Z.; Xiao, J.-A.; Xiang, H.-Y.; Chen, X.-Q.; Yang, H. Angew. Chem., Int. Ed. 2020, 59, 6706.

[39] Dai, W.; Geib, S. J.; Curran, D. P. J. Am. Chem. Soc. 2020, 142, 6261

[40] (a) Feldman, K. S.; Romanelli, A. L.; Ruckle, R. E.; Miller, R. F. J. Am. Chem. Soc. 1988, 110, 3300.

(b) Feldman, K. S.; Simpson, R. E. J. Am. Chem. Soc. 1989, 111, 4878

(c) Miura, K.; Fugami, K.; Oshima, K.; Utimoto, K. Tetrahedron Lett. 1988, 29, 5135.

(d) Feldman, K. S.; Romanelli, A. L.; Ruckle, R. E.; Jean, G. J. Org Chem. 1992, 57, 100.

(e) Feldman, K. S.; Berven, H. M.; Weinreb, P. H. J. Am. Chem. Soc. 1993, 115, 11364

(f) Journet, M.; Rouillard, A.; Cai, D.; Larsen, R. D. J. Org. Chem. 1997, 62, 8630.

(g) Feldman, K. S.; Fisher, T. E. Tetrahedron 1989, 45, 2969.

(h) Kim, S.; Lee, S. Tetrahedron Lett. 1991, 32, 6575.

(i) Zhang, H.; Jeon, K. O.; Hay, E. B.; Geib, S. J.; Curran, D. P.;

LaPorte, M. G. Org. Lett. 2014, 16, 94. (k) Zhang, H.; Curran, D. P. J. Am. Chem. Soc. 2011, 133, 10376

[41] Hashimoto, T.; Kawamata, Y.; Maruoka, K. Nat. Chem. 2014, 6, 702 .

[42] (a) Zhao, Q.-Q.; Zhou, X.-S.; Xu, S.-H.; Wu, Y.-L.; Xiao, W.-J.; Chen, J.-R. Org. Lett. 2020, 22, 2470

(b) Zhao, Q.-Q.; Chen J.; Zhou, X.-S.; Yu, X.-Y.; Chen, J.-R.; Xiao, W.-J. Chem.-Eur. J. 2019, 25, 8024 .

(c) Yu, X.-Y.; Zhao Q.-Q.; Chen, J.; Xiao, W.-J.; Chen, J.-R. Acc. Chem. Res. 2020, 53, 1066

[43] Xu, A.-Q.; Zhang, F.-L.; Ye, T.; Yu, Z.-X.; Wang, Y.-F. CCS Chem. 2019, 1,504 .

[44] (a) Kuivila, H. G. Acc. Chem. Res. 1968, 1, 299.

(b) Neumann, W. P. Synthesis 1987, 665.

[45] (a) Boyer, I. J. Toxicology 1989, 55, 253.

(b) Ingham, R. K., Rosenberg, S. D., Gilman, H. Chem. Rev. 1960 60,459 .

[46] (a) Hurd, R. N.; DeLaMater, G. Chem. Rev. 1961, 61, 45.

(b) Guo, W.-S.; Wen, L.-R.; Li, M. Org. Biomol. Chem. 2015, 13, 1942.

(c) Jagodziński, T. S. Chem. Rev. 2003, 103, 197.

[47] Wertheim, E. J. Am. Chem. Soc. 1935, 57, 545.

[48] Yu, Y.-J.; Zhang, F.-L.; Cheng, J.; Hei, J.-H.; Deng, W.-T.; Wang, Y.-F. Org. Lett. 2018, 20, 24.

[49] Du, W.; Curran, D. P. Org. Lett. 2003, 5, 1765.

[50] (a) Wang, J.; Qin, T., Chen, T.-G.; Wimmer, L.; Edwards, J. T.; Cornella, J.; Vokits, B.; Shaw, S. A.; Baran, P. S. Angew. Chem., Int. Ed. 2016, 55, 9676.

(b) Toriyama, F.; Cornella, J.; Wimmer, L.; Chen, T.-G.; Dixon, D. D; Creech, G.; Baran, P. S. J. Am. Chem. Soc. 2016, 138, 11132.

(c) Qin, T.; Cornella, J.; Li, C.; Malins, L. R.; Edwards, J. T.; Kawamura, S.; Maxwell, B. D.; Eastgate, M. D.; Baran, P. S. Science 2016, 352, 801.

(d) Qin, T.; Malins, L. R.; Edwards, J. T.; Merchant, R. R.; Novak, A. J. E.; Zhong, J. Z.; Mills, R. B.; Yan, M.; Yuan, C.; Eastgate, M. D.; Baran, P. S. Angew. Chem., Int. Ed. 2017, 56, 260.

(e) Huihui, K. M. M.; Caputo, J. A.; Melchor, Z.; Olivares, A. M.; Spiewak, A. M.; Johnson, K. A.; DiBenedetto, T. A.; Kim, S.; Ackerman, L. K. G.; Weix, D. J. J. Am. Chem. Soc. 2016, 138 5016.

(f) Lackner, G. L.; Quasdorf, K. W.; Overman, L. E. J. Am. Chem. Soc. 2013, 135, 15342.

(g) Lackner, G. L.; Quasdorf, K. W.; Pratsch, G.; Overman, L. E. J. Org. Chem. 2015, 80, 6012.

(h) Slutskyy, Y.; Overman, L. E. Org. Lett. 2016, 18, 2564.

(i) Huang, L.; Olivares, A. M.; Weix, D. J. Angew. Chem., Int. Ed. 2017, 56, 11901 .

(j) Tlahuext-Aca, A.; Garza-Sanchez, R. A.; Glorius, F. Angew. Chem., Int. Ed. 2017, 56, 3708.

(k) Kachkovskyi, G.; Faderl, C.; Reiser, O. Adv. Synth. Catal. 2013, $355,2240$.

(1) Jiang, M.; Yang, H.; Fu, H. Org. Lett. 2016, 18, 1968.

(m) Candish, L.; Teders, M.; Glorius, F. J. Am. Chem. Soc. 2017, 139, 7440 .

(n) Fawcett, A.; Pradeilles, J.; Wang, Y.; Mutsuga, T.; Myers, E. L.; Aggarwal, V. K. Science 2017, 357, 283.

(o) Murarka, S. Adv. Synth. Catal. 2018, 360, 1735.

[51] Lu, X.; Xiao, B.; Liu, L.; Fu, Y. Chem. Eur. J. 2016, 22, 11161.

[52] Jin J.-K.; Zhang F.-L.; Wang Y.-F. Acta Chim. Sinica 2019, 77, 889 (in Chinese).

(靳继康, 张风莲, 汪义丰, 化学学报, 2019, 77, 889.)

[53] Gao, L.; Wang, G.; Cao, J.; Yuan, D.; Xu, C.; Guo, X.; Li, S. Chem. Commun. 2018, 54, 11534 . 